\title{
Functional recovery after the systemic administration of mesenchymal stem cells in a rat model of neonatal hypoxia-ischemia
}

\author{
Takuro Sakai, MD, PhD, ${ }^{1,2}$ Masanori Sasaki, MD, PhD, ${ }^{2,4,5}$ Yuko Kataoka-Sasaki, MD, PhD, ${ }^{2}$ \\ Shinichi Oka, MD, PhD, ${ }^{2}$ Masahito Nakazaki, MD, PhD, ${ }^{2}$ Shinobu Fukumura, MD, PhD, ${ }^{1,2}$ \\ Masaki Kobayashi, MD, ${ }^{2,3}$ Hiroyuki Tsutsumi, MD, PhD, ${ }^{1}$ Jeffery D. Kocsis, PhD, ${ }^{4,5}$ and \\ Osamu Honmou, MD, PhD2,4,5
}

\begin{abstract}
'Department of Pediatrics, ${ }^{2}$ Department of Neural Regenerative Medicine, Research Institute for Frontier Medicine, and ${ }^{3}$ Department of Perinatal Medicine, Sapporo Medical University School of Medicine, Sapporo, Japan; "Department of Neurology, Yale University School of Medicine, New Haven; and ${ }^{5}$ Center for Neuroscience and Regeneration Research, VA Connecticut Healthcare System, West Haven, Connecticut
\end{abstract}

OBJECTIVE Children who have experienced neonatal hypoxic-ischemic encephalopathy often develop cerebral palsy. Although many treatments have been performed, few effective therapies are available. In this study, the authors tested in rats with hypoxia-ischemia (HI) injuries the hypothesis that the systemic infusion of mesenchymal stem cells (MSCs) would result in functional improvement by facilitating neural compensation in the contralesional cortex.

METHODS Postnatal day (P) 7 (P7) rats that had undergone unilateral hemisphere hypoxia-ischemia (modified RiceVannucci model) were randomly assigned to MSC-infused or vehicle-infused groups. MSCs $\left(1.0 \times 10^{6} / 200 \mu \mathrm{L}\right)$ or vehicle were intravenously infused on P10. Brain volume was measured using in vivo MRI on P8 and P35. On P35, the rats were sacrificed after their behavior was evaluated using a beam walk test, and their brains were then prepared for histological analyses.

RESULTS The MSC-treated group had fewer slips on the beam walk test compared to those in the vehicle group ( $p=$ 0.041). MRI was used to measure the volumes of the whole brain, contralesional brain (hemisphere), and residual brain regions of interest, and the results indicated increased brain volume after the intravenous MSC infusions. The histological analyses revealed increased thicknesses of the contralesional cortex and corpus callosum in the MSC group compared with those in the vehicle group $(p=0.021, p=0.019)$, which confirmed the volume increases. In the contralesional cortex, the MSC-treated group exhibited significant increases in the numbers of NeuN-positive cells $(p=0.004)$ and synaptic puncta $(p=0.000)$ compared with the numbers observed in the vehicle group.

CONCLUSIONS The intravenous infusion of MSCs resulted in improvements in functional outcome, increased brain volume, and enhanced synaptogenesis in $\mathrm{HI}$ rats.

https://thejns.org/doi/abs/10.3171/2018.5.PEDS1845

KEYWORDS hypoxic ischemia; cerebral palsy; mesenchymal stem cells; congenital

$\mathrm{C}$ EREBRAL palsy (CP), which is the most common cause of motor disabilities in children, has a prevalence of 1.7-3.1 per 1000 live births in high-income countries and a higher prevalence in low-income countries. ${ }^{11}$ Because few established treatments are available for most children with $\mathrm{CP}$, the development of effective therapies for these children has become a priority. ${ }^{24}$ Children with neonatal hypoxic-ischemic encephalopathy often develop CP. A hypoxia-ischemia (HI) insult can trigger prolonged ischemia-induced injury mechanisms that lead to progressive brain damage and persistent motor dysfunction. ${ }^{20}$

ABBREVIATIONS $\mathrm{CP}=$ cerebral palsy; $\mathrm{GFP}=$ green fluorescent protein; $\mathrm{HI}=$ hypoxia-ischemia; $\mathrm{i} . \mathrm{p}$. = intraperitoneal; $\mathrm{MSC}=$ mesenchymal stem cell; $\mathrm{P}=$ postnatal day; PBS = phosphate-buffered saline; $S E M=$ standard error of the mean.

SUBMITTED January 22, 2018. ACCEPTED May 10, 2018.

INCLUDE WHEN CITING Published online August 3, 2018; DOI: 10.3171/2018.5.PEDS1845. 
The results of previous studies using HI models have suggested the therapeutic efficacy of intravenous infusions of mesenchymal stem cells (MSCs) that were derived from bone marrow ${ }^{5,26}$ and umbilical cord blood cells. ${ }^{1,2}$ The mechanisms underlying the therapeutic efficacy of these cellular treatments might include neuroprotection through neurotrophic factors. ${ }^{27}$

Functional compensation that increases activity after a stroke might result from enhancement of the remaining and newly developed synaptic circuits. However, few studies have examined the neuronal basis of the compensatory mechanisms that occur following neonatal HI brain injury. ${ }^{13,22,23}$ Unilateral ischemic animal models, such as the Rice-Vannucci model, can be used to compare lesioned and unlesioned cerebral hemispheres after the intravenous infusions of MSCs.

In this study, we tested the hypothesis that systemically infused MSCs would improve functional recovery through neural compensation by enhancing neural plasticity in the contralesional cortex in a rat model of HI. Behavioral outcome evaluations, in vivo MRI, and histological analyses were performed after the MSC infusions.

\section{Methods \\ Animals}

The use of the animals in this study was approved by the animal care and use committee of Sapporo Medical University, and all procedures were conducted in accordance with institutional guidelines.

\section{HI Model}

In this study, we used the Rice-Vannucci model with minor modifications as the HI model. ${ }^{18,20}$ On postnatal day (P) 7 (P7), the animals (Sprague Dawley rats, weight range 13-17 g) were anesthetized with an intraperitoneal (i.p.) injection of ketamine $(25 \mathrm{mg} / \mathrm{kg})$ and xylazine $(5 \mathrm{mg} /$ $\mathrm{kg}$ ). The left common carotid artery, which was identified through a longitudinal neck incision in the midline, was isolated from the vagus nerve, permanently double-ligated with a 6-0 surgical silk suture, and sectioned between the double-ligatures. The entire surgical procedure was completed within 10 minutes. After the wounds were sutured, the animals were returned to their cages and allowed to recover for 1 hour in the company of their mothers. The rats were then placed in a hypoxia chamber with $8 \%$ oxygen balanced with nitrogen for 2 hours. The hypoxia chamber contained a temperature-control mat to maintain the ambient temperature inside the chamber at a normal range $\left(37^{\circ} \mathrm{C}\right)$. After the hypoxic exposure, the pups were returned to their mothers for recovery. Most of these pups showed small ischemic volume on MRI without obvious neurological deficits and did not meet our inclusion criteria. Therefore, we only included animals displaying obvious neurological deficits who met the criteria used to standardize the ischemic volume $\left(120-180 \mathrm{~mm}^{3}\right)$ at $\mathrm{P} 8$. We used a total of 280 pups and the inclusion rate was 5\%. The use of MRI to screen for a standardized lesion volume prior to randomization and treatment provided us with an efficient protocol to minimize animal use and maximize the observation of potential effectiveness of MSCs in this model system.

\section{Preparation of MSCs From Rat Bone Marrow}

The MSC culture methods were based on our previously described methods. ${ }^{10,12,14}$ Briefly, bone marrow was obtained from the femoral bones of adult (6- to 8-weekold) Sprague Dawley rats or green fluorescent protein (GFP)-expressing rats [W-Tg(CAG-GFP)184Ys] and diluted to $15 \mathrm{~mL}$ with Dulbecco's modified Eagle's medium (DMEM) (Sigma-Aldrich Corp.) that was supplemented with $10 \%$ heat-inactivated fetal bovine serum (Thermo Fisher Scientific Inc.,), 2 mM L-glutamine (Sigma-Aldrich Corp.), $100 \mathrm{U} / \mathrm{mL}$ of penicillin, and $0.1 \mathrm{mg} / \mathrm{mL}$ of streptomycin (Thermo Fisher Scientific Inc.) and then incubated for 3 days $\left(5 \% \mathrm{CO}_{2}, 37^{\circ} \mathrm{C}\right)$. When the cultures had almost reached confluence, the adherent cells were detached with trypsin-ethylenediaminetetraacetic acid solution (SigmaAldrich Corp.) and subcultured at a density of $1 \times 10^{4}$ cells $/ \mathrm{mL}$. In the present study, we used the MSCs after 3 passages. A previous phenotypic analysis of the surface antigens on the MSCs revealed $\mathrm{CD}_{45}^{-}, \mathrm{CD}^{2} 3^{+}, \mathrm{CD} 90^{+}$, and $\mathrm{CD} 106^{-}{ }^{-}$?

\section{MSC Infusion Procedures}

We employed 3 groups in this study, HI rats with MSCs (MSC group, $n=4$ ), HI rats without MSCs (vehicle group, $\mathrm{n}=5$ ), and an age-matched intact (nonlesioned) control group $(n=5)$. The HI rat pups were randomly assigned to the vehicle $(n=5)$ or MSC $(n=4)$ experimental groups. The rats in the vehicle group were injected intravenously with fresh DMEM (vehicle) alone through the femoral vein (without donor cell administration) at P10. We confirmed high cell viability (>99\%) with $0.4 \%$ trypan blue from cell suspension aliquots taken from the injection cell suspensions immediately after the in vivo infusion. The rats in the MSC group were injected intravenously with MSCs $\left(1.0 \times 10^{6}\right.$ cells $)$ in fresh DMEM $(200 \mu \mathrm{L})$ at P10. All rats, including age-matched intact (unlesioned) control rats $(n=5)$, were injected every other day with cyclosporine $\mathrm{A}(10 \mathrm{mg} / \mathrm{kg}$, i.p.). The sex distribution was as follows: MSC group, 1 female and 3 male rats; vehicle group, 2 female and 3 male rats; and control group, 2 female and 3 male rats.

\section{Motor Function Test}

The beam walk test was performed at P35 (25 days after the MSC or vehicle infusions) as described in a previous report. ${ }^{9}$ A wooden beam $(100 \mathrm{~cm}$ long $\times 15 \mathrm{~mm}$ wide) was elevated $40 \mathrm{~cm}$ above the floor. The home cage of each rat was positioned at one end of the beam. The rats were placed on the far end of the beam and trained to walk the beam toward their home cage. To quantify motor deficits, the number of sideslips of the hind foot from the beam in 3 trials with 15-minute intermittent recovery periods were recorded. If a rat was not able to walk on the beam without falling, the number of sideslips was set to 10. The mean values for the 3 trials were used to calculate the group means.

\section{In Vivo MRI Studies}

The rats were anesthetized with ketamine $(25-50 \mathrm{mg} /$ $\mathrm{kg}$, i.p.) and xylazine $(5-10 \mathrm{mg} / \mathrm{kg}$, i.p.). Each rat was 
placed in an animal holder/MRI probe apparatus and positioned inside the magnet so that the animal's head remained motionless inside the imaging coil. All MRI measurements were performed using a 7-T $18-\mathrm{cm}$-bore superconducting magnet (Oxford Instruments plc) that was interfaced with a Unity INOVA console (Varian Medical Systems, Inc.) as previously described. ${ }^{4,8} \mathrm{~T} 2$-weighted images were obtained from $1.0-\mathrm{mm}$-thick coronal sections with $0.5-\mathrm{mm}$ gaps using a $30 \mathrm{~mm} \times 30 \mathrm{~mm}$ field of view (TR $3000 \mathrm{msec}$, TE $30 \mathrm{msec}$ ) and reconstructed using a $256 \times 256$ image matrix. Accurate positioning of the brain was ensured by centering the slice that was $5 \mathrm{~mm}$ posterior to the rhinal fissure with the head of the rat held in a flat skull position.

T2-weighted images were obtained at P8 and P35. The animals that did not meet the criteria used to standardize the initial ischemic volume $\left(120-180 \mathrm{~mm}^{3}\right)$ at P8 (day after the HI injury) were excluded. The remaining rats were randomized to the 2 experimental groups.

Brain volume was calculated from the MR images using imaging software (Scion Image, Version Beta 4.0.2; Scion Corporation). Brain volume $\left(\mathrm{mm}^{3}\right)$ was determined on 9 serial coronal images of the cerebrum. Brain volume was calculated with consideration of slice thickness (1 $\mathrm{mm}$ /slice). The boundaries of the "whole brain," "contralesional brain hemisphere," and "residual brain" regions of interest (ROIs) were demarcated by raters who were experienced in neuroanatomy and neuroradiology. The "whole brain" ROI contained the intracranial cerebrum volume. To measure the "residual brain" volume, the edges of the lesions where the signal intensity was at least 1.25 times that of the counterpart in the contralesional brain lesion after adjusting optimally for contrast were traced, as previously described ${ }^{21}$ Thus, the "residual brain" volume was calculated as the "whole brain" volume minus the volume of the ischemic tissue with higher signal intensities. The ROIs outlined above were then summed and multiplied by the slice thickness plus the interslice gap to calculate the "whole brain," "contralesional brain hemisphere," and "residual brain" volumes.

\section{Immunohistological Analysis}

The rats were deeply anesthetized with ketamine and xylazine $(50 / 10 \mathrm{mg} / \mathrm{kg}$, i.p.) and perfused with saline and $0.1-\mathrm{M}$ phosphate buffer, and the brain was removed and then processed for standard frozen sectioning. Cryosections were cut using a cryostat and mounted on glass slides. The sections were washed in phosphate-buffered saline (PBS) and $0.1 \%$ Tween 20 three times, blocked in $10 \%$ normal goat serum and $0.3 \%$ Triton X-100 in PBS at room temperature for 60 minutes, and then incubated overnight in primary antibodies that were diluted with $5 \%$ normal goat serum, $0.3 \%$ Triton $\mathrm{X}-100$, and $\mathrm{PBS}$ at $4^{\circ} \mathrm{C}$. The 50- $\mu$ m-thick cryosections were immunolabeled using a mouse antineuronal nuclei (NeuN) antibody (1:1000, MAB377; MilliporeSigma) as a neuronal marker, rabbit polyclonal antiglial fibrillary acidic protein (GFAP) antibody (1:1000, ab7260, Abcam plc) as a glial cell marker, rabbit anti-synaptophysin antibody (1:1000 ab52636; Abcam plc) as a presynaptic vesicle protein marker and indicator of presynaptic plasticity and synaptogenesis, and

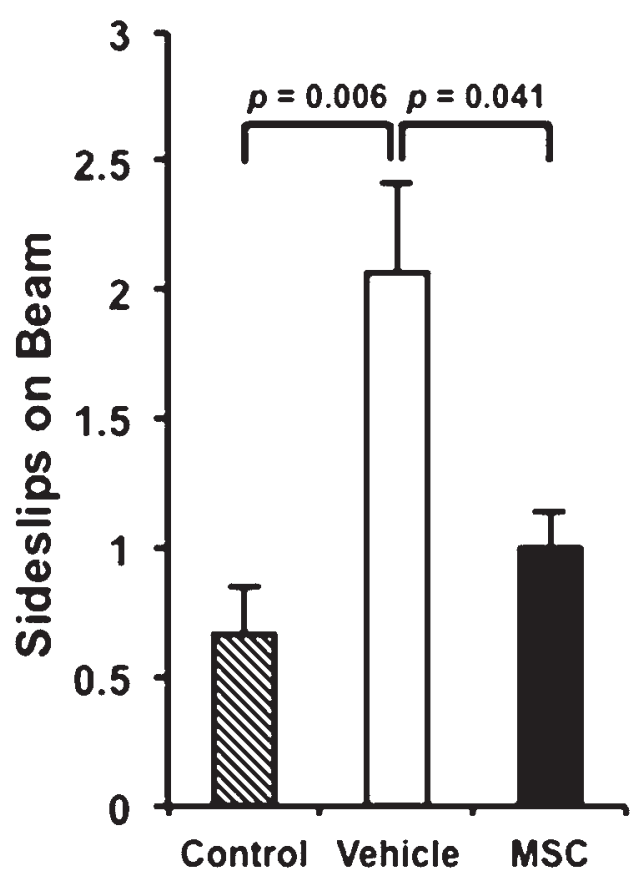

FIG. 1. Functional improvement in the MSC group on the beam walk test. The results of the beam walk test demonstrated that the MSCtreated animals displayed better functional outcomes compared to those in the vehicle group at P35. The mean number of sideslips on the beam was significantly decreased in the MSC group compared with the vehicle group. Error bars indicate SEM.

chicken polyclonal anti-GFP antibody (1:1,000, ab13970; Abcam plc) to detect GFP. After washing in PBS and $0.1 \%$ Tween 20 four times, the sections were incubated in the appropriate secondary antibody [NeuN: goat antimouse Alexa Fluor 594 immunoglobulin G (IgG; 1:500, Jackson 115-585-146; Jackson ImmunoResearch Laboratories, Inc.); GFAP and synaptophysin: goat anti-rabbit Alexa Fluor 488 IgG conjugate (1:500, A-11034; Thermo Fisher Scientific Inc.), and GFP: goat anti-chicken Alexa Fluor 488 H \& L immunoglobulin Y pre-adsorbed conjugate $(1: 1000,150173$; Abcam plc)], counterstained with 4',6-diamidino-2-phenylindole (DAPI), and cover slipped with VECTASHIELD (Vector Laboratories, Inc.). The frozen sections were examined using a confocal microscopy (excitation/emission: $405 \mathrm{~nm} / 410-502 \mathrm{~nm}$ for DAPI; $488 \mathrm{~nm} / 499-553 \mathrm{~nm}$ for GFAP, synaptophysin, and GFP; and $561 \mathrm{~nm} / 499-553 \mathrm{~nm}$ for NeuN; LSM 780 ELYRA S.1 system; Carl Zeiss AG).

\section{Quantitative Analysis}

For the quantification, $\mathrm{NeuN}^{+}$cells were considered to represent neurons, while $\mathrm{GFAP}^{+}$cells were considered to represent glial cells. Unbiased stereological analyses were performed using a light microscope (BX51; Olympus Corporation) that was equipped with an automatic stage and coupled to a computer running Stereo Investigator software (MBF Bioscience). One in every 20 serial sections $(50-\mu \mathrm{m}$-thick) was used in the analysis. The contours were drawn around the M1 cortical area, and cell counting was performed on slices at the bregma- $3.3 \mathrm{~mm}$ 
Whole Brain

Control Vehicle

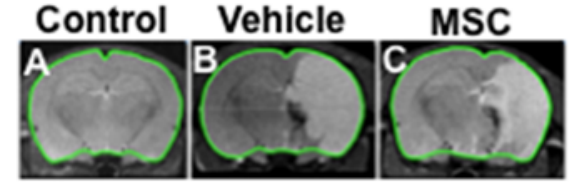

I

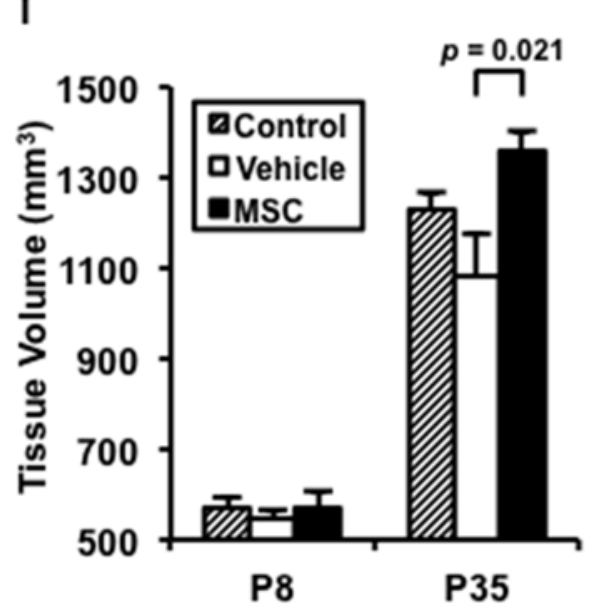

Contralesional Brain Hemisphere
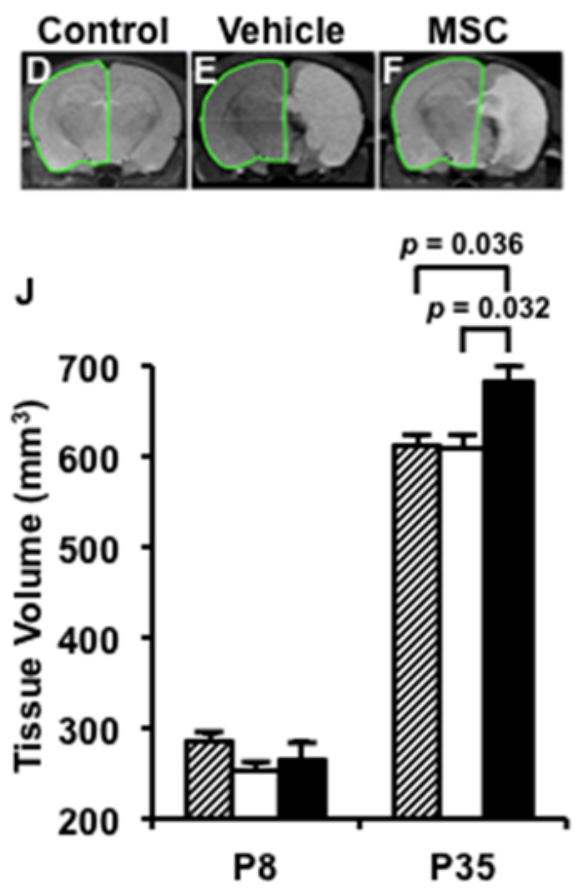

Residual Brain
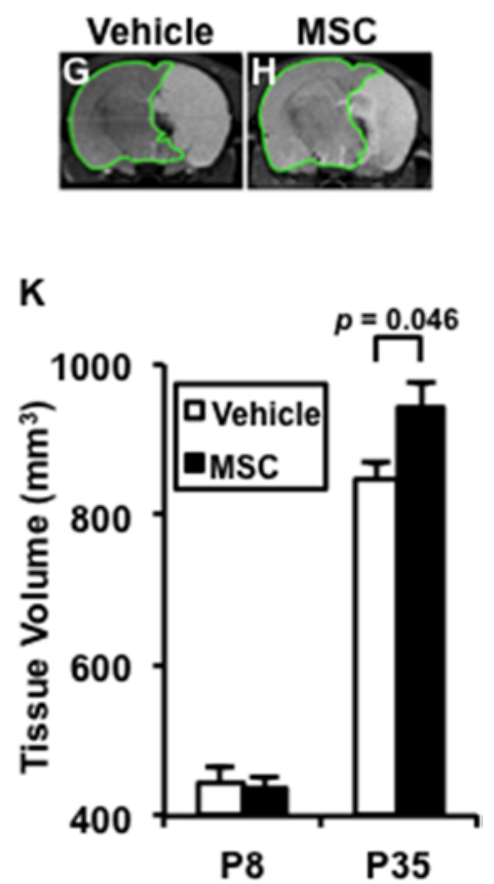

FIG. 2. MRI analysis of brain volume in the MSC group at P35. The boundaries of the "whole brain," "contralesional brain hemisphere," and "residual brain" ROls were traced to analyze brain volume at postnatal day 35 (P35). A-H: Representative T2-weighted images and the boundaries of the regions of interest are shown for the "whole brain" in the control group (A), vehicle group (B), and MSC group (C); the "contralesional brain hemisphere" in the control group (D), vehicle group (E), and MSC group (F); and the "residual brain" in the vehicle group $(\mathrm{G})$ and MSC group $(\mathrm{H})$. I-K: Comparison of "whole brain" (I), "contralesional brain hemisphere" (J), and "residual brain" $(\mathrm{K})$ volumes in the control, vehicle, and MSC groups at P8 and P35. The mean volumes in the MSC group were significantly larger than those in the vehicle group at P35. The ischemic volumes did not differ significantly between the groups. Error bars indicate SEM. Please note that the control group does not have residual brain images because they do not have ischemic lesions.

to the bregma-1.3 mm level (modified from Paxinos and Watson, 1998 ${ }^{16}$ ). To estimate the number of neurons and glial cells in the cortex, the optical fractionator method was used to count the cells in a 3D counting frame $(100 \times$ $100 \times 10 \mu \mathrm{m})$ using a sampling grid $(250 \times 250 \mu \mathrm{m})$. The average Schaffer coefficient of error for cell counting was $0.04-0.09 .{ }^{25}$

For the quantitative analysis of synaptophysin, ${ }^{21}$ confocal images were collected from layer III/IV in the contralesional motor cortex according to the rat stereotaxic atlas (bregma - $3.3 \mathrm{~mm}$; modified from Paxinos and Watson ${ }^{16}$ ). The levels of synaptophysin were quantified in the ROIs $(1024 \times 1024$ pixels). The coordinates of the center of the ROI relative to bregma were $-3.3 \mathrm{~mm}$ caudal, $3 \mathrm{~mm}$ lateral, and $0.5 \mathrm{~mm}$ deep. The gain thresholds and amplitude offsets were kept constant for the motor cortex imaging in the contralesional areas in each section. The synaptic puncta were counted in each field using ImageJ Analysis Software (version 1.48; https://imagej.nih.gov/ij/).

\section{Statistical Analysis}

All data were analyzed using SPSS version 22 (IBM Corp.). The differences among the groups were analyzed using one-way analyses of variance followed by Scheffé post hoc tests. The differences in the volumes of the residual brain between the 2 groups were compared using
Student t-tests. A p value less than 0.05 was considered statistically significant. The data are presented as the mean \pm the standard error of the mean (SEM).

\section{Results}

\section{Behavioral Analysis}

To assess the rats' fine motor coordination and balance, the beam walk test was performed at P35. As shown in Fig. 1, the mean number of sideslips was significantly decreased $(\mathrm{p}=0.041)$ in the MSC group $(1.00 \pm 0.14)$ compared with the vehicle group $(2.07 \pm 0.34)$. The mean number of slips in the control group was $0.67 \pm 0.18$, which did not differ significantly from the number for the MSC group. These results suggested that the intravenous infusion of MSCs improved behavioral function.

\section{MRI Characterization of Brain Volume}

To investigate the effects of intravenous infusion of MSCs on HI rats, we analyzed brain volume focusing on the "whole brain," "contralesional brain hemisphere," and "residual brain," as illustrated in Fig. 2, using in vivo MRI (Fig. 2; $n=4$, MSC group; $n=5$, vehicle group; $n=5$, control group). T2-weighted images were obtained at P8 and confirmed no difference with respect to initial ischemic volume between the MSC and vehicle groups (vehicle 

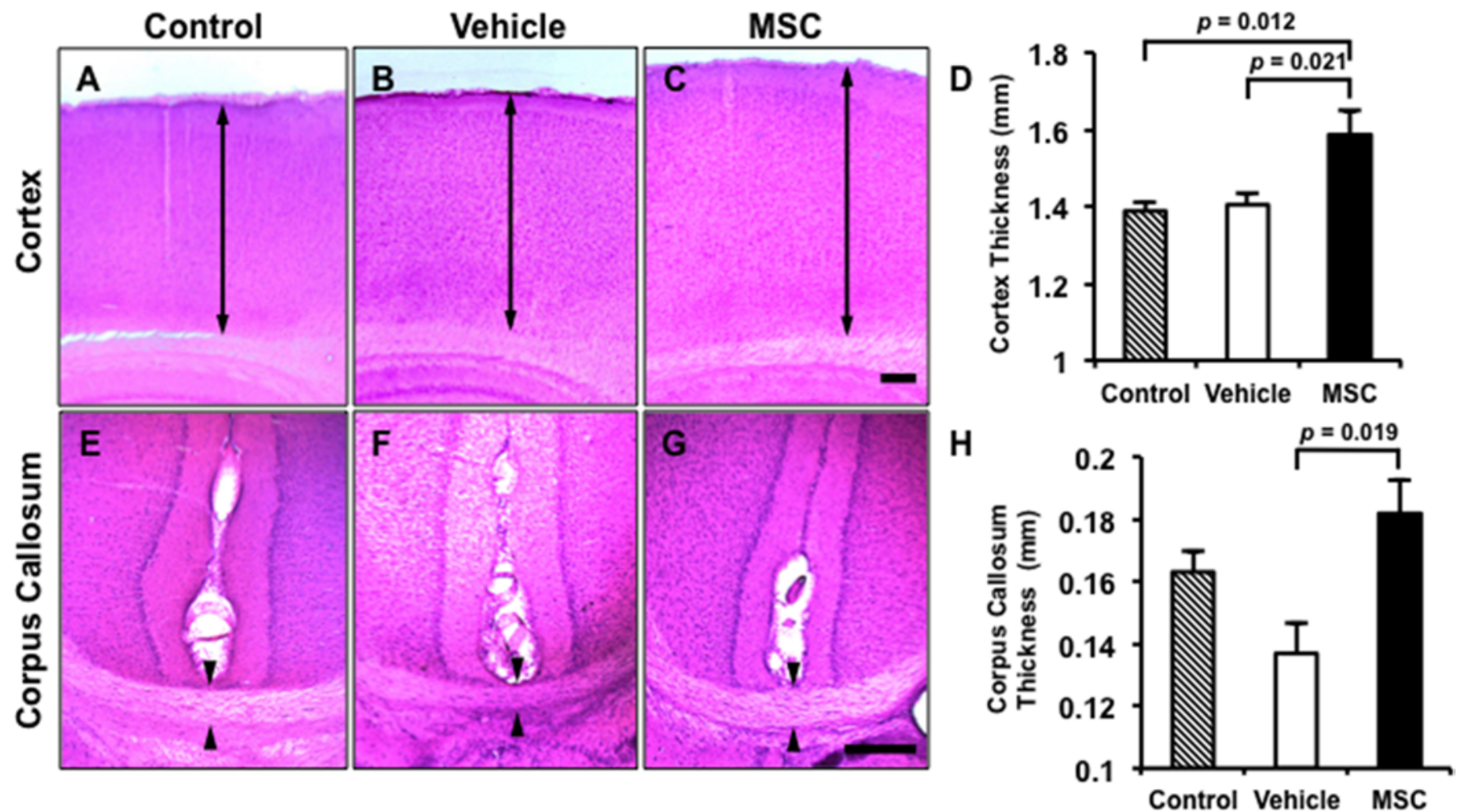

FIG. 3. Results of histopathological analysis showing increased thickness of both the contralesional cortex and corpus callosum in the MSC group. A-C: Hematoxylin and eosin ( $\mathrm{H} \& \mathrm{E}$ )-stained sections demonstrating the thickness (as indicated by the doubleheaded arrows) of the contralateral cortex in representative specimens from the control (A), vehicle (B), and MSC (C) groups. D: Quantification results showing that the mean cortical thickness in the contralesional brain was significantly greater in the MSC group than in the control or vehicle group. E-G: H \& E-stained sections demonstrated the thickness (as indicated by the arrowheads) of the corpus callosum in the control (E), vehicle (F), and MSC $(G)$ groups. H: Quantification results showing that the mean thickness of the corpus callosum was significantly greater in the MSC group than in the vehicle group. Bar $=200 \mu \mathrm{m}(\mathrm{A}-\mathrm{C}$ and $\mathrm{E}-\mathrm{G})$.

group, $143.6 \pm 8.0 \mathrm{~mm}^{3}$; MSC group, $155.7 \pm 4.9 \mathrm{~mm}^{3} ; \mathrm{p}$ $=0.266$ ). At P35, the mean volume of "whole brain" in the control group was $1227.5 \pm 43.3 \mathrm{~mm}^{3}$ (Fig. 2A and I). The mean volume in the MSC group (Fig. 2C; $1358.3 \pm$ $90.9 \mathrm{~mm}^{3}$ ) was larger than that of the vehicle group (Fig. $2 \mathrm{~B} ; 1083.3 \pm 37.5 \mathrm{~mm}^{3}$ ) (Fig. 2I, $\mathrm{p}=0.021$ ). The mean volume of the "contralesional brain hemisphere" in the control group was $610.6 \pm 21.0 \mathrm{~mm}^{3}$ (Fig. 2D and J), and the mean volume in the MSC group (Fig. 2F; $683.3 \pm 14.5$ $\mathrm{mm}^{3}$ ) was larger than in the vehicle group (Fig. 2E; 609.0 $\pm 11.6 \mathrm{~mm}^{3}$ ) or control group (Fig. 2D) (Fig. 2J; $\mathrm{p}=0.032$, $\mathrm{p}=0.036$ ). The mean volume of "residual brain" ("whole brain" minus "ischemic tissue volume") in the MSC group (Fig. $2 \mathrm{H} ; 941.3 \pm 34.4 \mathrm{~mm}^{3}$ ) was larger than that in the vehicle group (Fig. 2G; $847.1 \pm 21.7 \mathrm{~mm}^{3}$ ) (Fig. $2 \mathrm{~K} ; \mathrm{p}=$ 0.046). These results, which are summarized in Fig. 2I, J, and $\mathrm{K}$, respectively, suggest that infused MSCs induced the growth of brain volume in the nonaffected brain tissue of HI rats. Note that there was no significant difference in the ischemic volume (determined as higher-intensity areas) between the vehicle group $\left(236.2 \pm 38.0 \mathrm{~mm}^{3}\right)$ and the MSC group $\left(417.0 \pm 114.7 \mathrm{~mm}^{3}\right)$ at P35 $(\mathrm{p}=0.111)$.

\section{Cortex and Corpus Callosum Thickness}

Hematoxylin and eosin staining was performed to ex- amine brain morphological changes at P35 $(n=4$, MSC group; $n=5$, vehicle group; $n=5$, control group) (Fig. 3). The mean cortical thickness in contralesional brain in the control group was $1.388 \pm 0.022 \mathrm{~mm}$ (Fig. 3A). The mean thickness in contralesional brain was significantly greater in the MSC group (Fig. 3C; $1.587 \pm 0.062 \mathrm{~mm}$ ) than in the vehicle group (Fig. 3B; $1.404 \pm 0.029 \mathrm{~mm})(\mathrm{p}=0.012)$. The mean corpus callosum thickness in the control group was $0.1631 \pm 0.0068 \mathrm{~mm}$ (Fig. 3E). The corpus callosum thickness also was significantly greater in the MSC group (Fig. $3 \mathrm{G} ; 0.1816 \pm 0.0108 \mathrm{~mm}$ ) than in the vehicle group (Fig. $3 F ; 0.1369 \pm 0.0097 \mathrm{~mm})(p=0.019)$. These results are summarized in Fig. 3D and $\mathrm{H}$, respectively, and suggest that intravenous infusion of MSCs in the HI rats increased the thickness of cortex and corpus callosum.

\section{Quantification of Cortical Neuronal Cells}

To determine whether intravenous infusion of MSCs affects the neuronal profile on contralesional M1 cortex, immunohistochemical analysis was performed at P35 ( $\mathrm{n}=$ 4 , MSC group; $\mathrm{n}=5$, vehicle group; $\mathrm{n}=5$, control group). The mean density of $\mathrm{NeuN}^{+}$cells was significantly greater in the MSC-treated group (Fig. 4C and F; $13.39 \pm 0.86$ $\times 10^{4}$ cells $/ \mathrm{mm}^{3}$ ) than in the vehicle group (Fig. $4 \mathrm{~B}$ and E; $8.63 \pm 0.79 \times 10^{4}$ cells $/ \mathrm{mm}^{3}$ ) or control group (Fig. 4A 


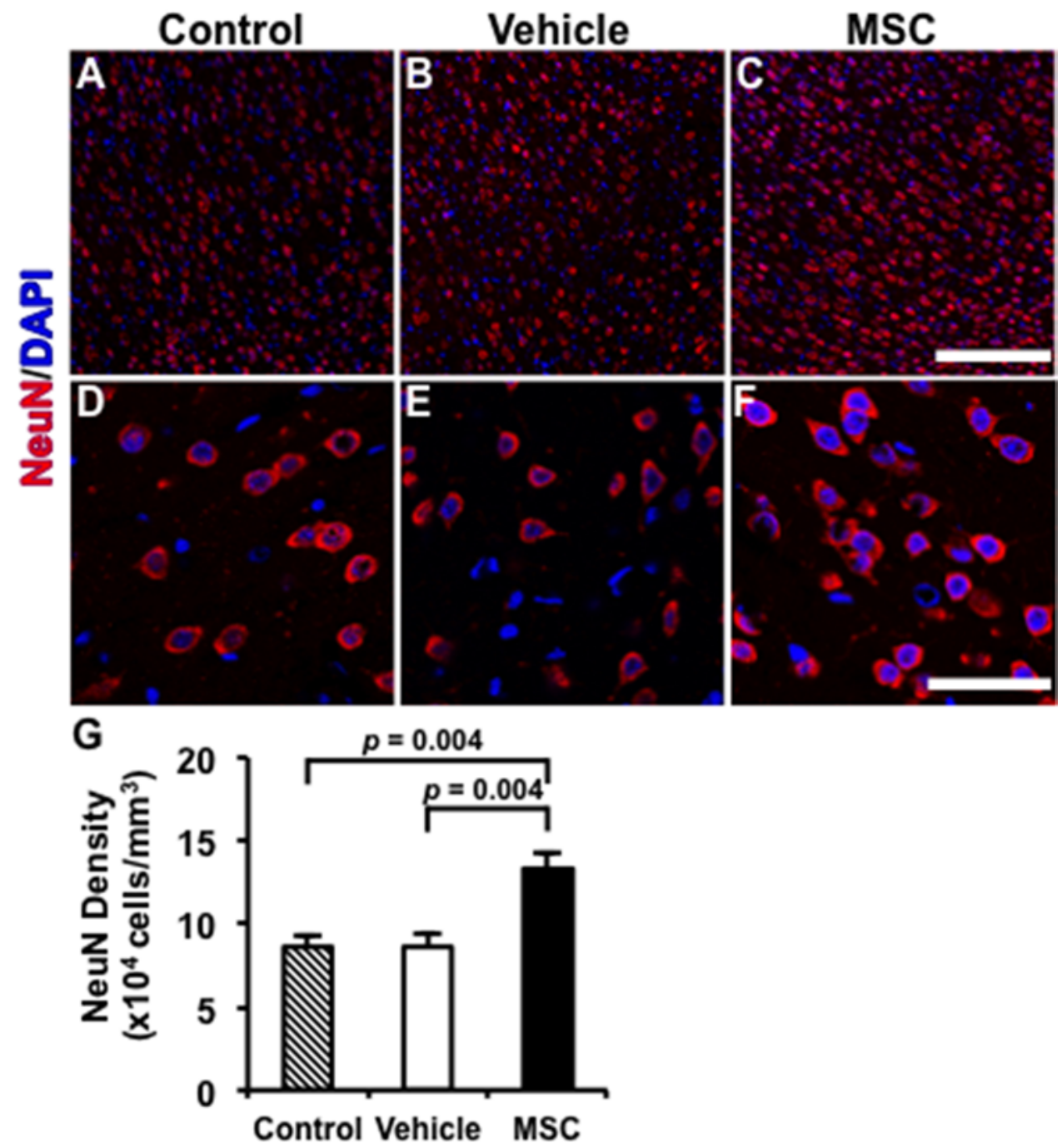

FIG. 4. Increased NeuN-positive cells in the cortex after MSC infusion. Immunohistological analysis demonstrated that the number of NeuN-positive cells (red) was increased in the specimens from the contralesional cortex in the MSC group ( $\mathbf{C}$ and $\mathbf{F}$ ) compared with corresponding specimens from the control $(A$ and $D)$ and vehicle ( $B$ and $E)$ groups (specimens counterstained with DAPI staining for nuclei [blue]). This finding was confirmed by quantification of the NeuN-positive neurons (G). Bar = $50 \mu \mathrm{m}(\mathrm{A}-\mathrm{C}), 200$ $\mu \mathrm{m}(\mathrm{D}-\mathrm{F})$.

and $\mathrm{D} ; 8.63 \pm 0.60 \times 10^{4}$ cells $\left./ \mathrm{mm}^{3}\right)(\mathrm{p}=0.004)($ Fig. $4 \mathrm{G})$. The mean density of GFAP ${ }^{+}$cells in the control group was $2.49 \pm 0.10 \times 10^{4}$ cells $/ \mathrm{mm}^{3}$ (Fig. 5A and D). There was no significant difference between the mean density of GFAP ${ }^{+}$ cells in the MSC group (Fig. 5C and F; $3.47 \pm 0.12 \times 10^{4}$ cells $/ \mathrm{mm}^{3}$ ) and the vehicle group (Fig. 5B and E; $3.35 \pm$ $0.23 \times 10^{4}$ cells $/ \mathrm{mm}^{3}$ ), although the number of GFAP ${ }^{+}$cells in these experimental groups was significantly higher than in the controls $(p=0.006, p=0.010)$. Collectively, these results suggested that the intravenous infusion of MSCs in the HI rats induced the growth of brain tissue, which increased the cellular components in the contralesional cortex.

\section{Quantification of Synaptic Density}

To determine whether infused MSCs induce synaptogenesis in the contralesional M1 cortex, synaptic density was examined at P35 $(\mathrm{n}=4$, MSC group; $\mathrm{n}=5$, vehicle group; $n=5$, control group). Synaptophysin was used to quantify synaptic density (Fig. 6A-C). The density of synaptic puncta in the MSC-treated group (Fig. 6C; $0.611 \pm$ 0.050 puncta $/ \mu^{2}$ ) was significantly greater than in the vehicle (Fig. 6B; $0.181 \pm 0.031$ puncta $/ \mu^{2}$ ) and control (Fig. 6A; $0.397 \pm 0.059$ puncta/ $\left.\mu^{2}{ }^{2}\right)$ groups $(p=0.000$, $\mathrm{p}=0.033$ ) (Fig. 6D), indicating that the MSC infusions enhanced synaptogenesis.

\section{Detection of GFP-MSCs}

The histological analyses of specimens obtained 3 days (at P13) after infusion of MSCs derived from the GFP-expressing rats (GFP-MSCs) indicated that the infused cells survived and were distributed to the contralesional cortex $(n=4)($ Fig. 7A). No green cells were observed after infusion of MSCs derived from wild type rats $(n=4)$ which 


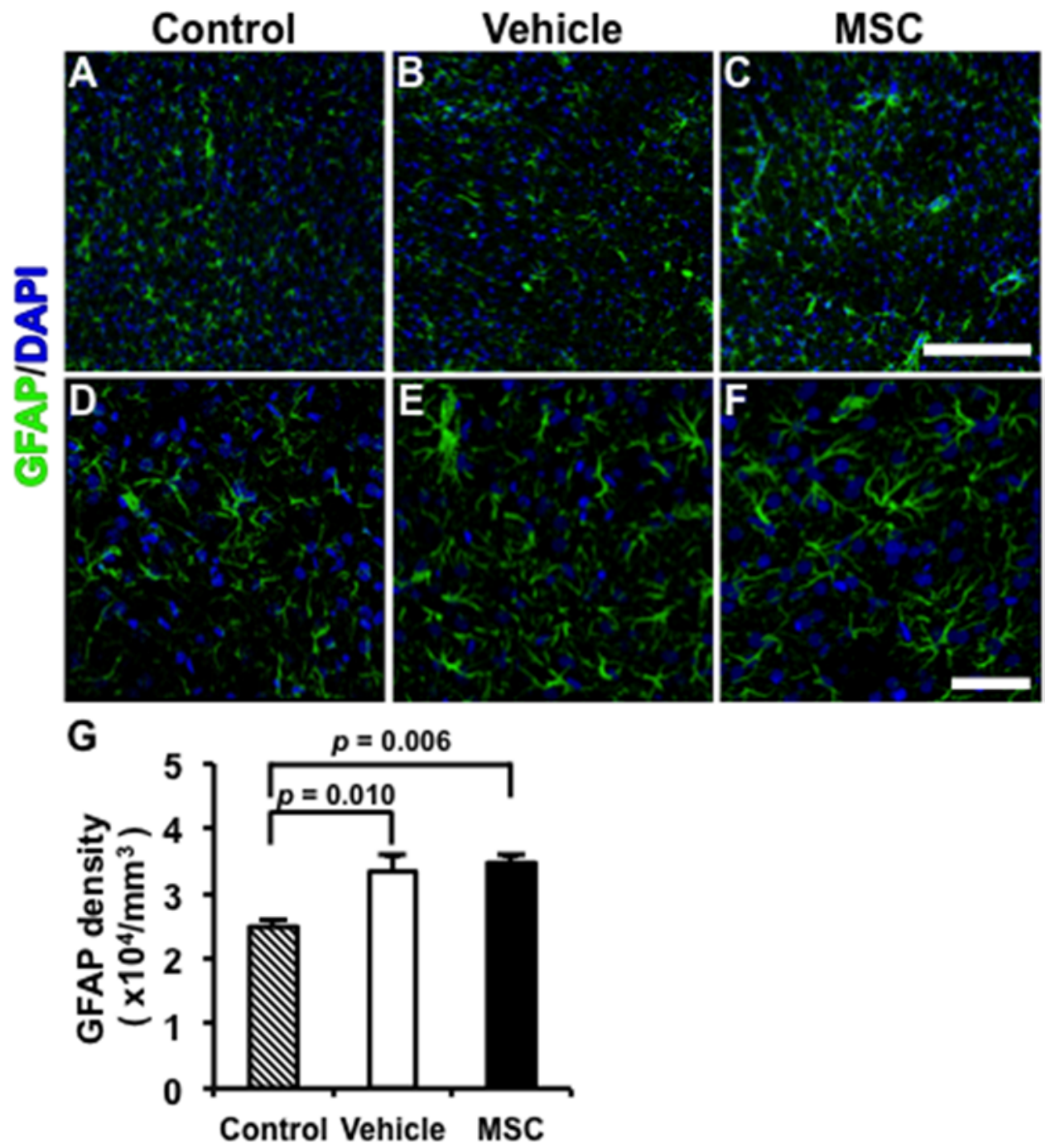

FIG. 5. GFAP-positive cells in the cortex after infusion. Immunohistological analysis demonstrated the number of GFAP-positive cells (green) in specimens from the contralesional cortex in the control (A and D), vehicle (B and E), and MSC groups (C and F) (specimens counterstained with DAPI staining for nuclei [blue]). Quantification of the GFAP-positive cells (G). Bar $=50 \mu \mathrm{m}(\mathrm{A}-\mathrm{C})$, $200 \mu \mathrm{m}(\mathrm{D}-\mathrm{F})$.

confirmed that GFP fluorescence signals were from the GFP-MSCs, not autofluorescence from endogenous tissue (Fig. 7B).

\section{Discussion}

The primary objective of this study was to elucidate the mechanisms underlying the therapeutic efficacy of intravenous infusion of MSCs in a rat model of HI. Compared to the control and vehicle-infused groups, the rats in the MSC group exhibited significant decreases in the number of sideslips in the beam walk testing paradigm, which was used to evaluate fine motor coordination and balance. These results suggested that the infused MSCs facilitated functional improvement. The MRI analyses demonstrated that the volumes of the "whole brain," "contralesional brain hemisphere," and "residual brain" in the MSC group were increased compared with those in the vehicle group at P35. Histological examination confirmed that the thicknesses of both the contralesional cortex and corpus callosum were increased in the MSC group compared with the vehicle group. The number of $\mathrm{NeuN}^{+}$neurons was increased in the contralesional cortex of the MSC-infused animals compared with the vehicle group. The intravenous infusion of MSCs also facilitated synaptogenesis in the contralesional cortex, as the density of synaptic puncta was increased in the MSC group compared with the vehicle group. In the present study, many clearly identified GFP-MSCs were detected in the contralesional cortex of the MSC-treated rats. The acute accumulation of the infused GFP-MSCs in the cortex might provide therapeutic efficacy through various mechanisms..$^{2,15,21}$

In this study, we focused on elucidating the neuronal bases of the compensatory mechanisms that occur after intravenous infusion of MSCs in a rat model of HI. The "whole brain," "contralesional brain hemisphere," and 

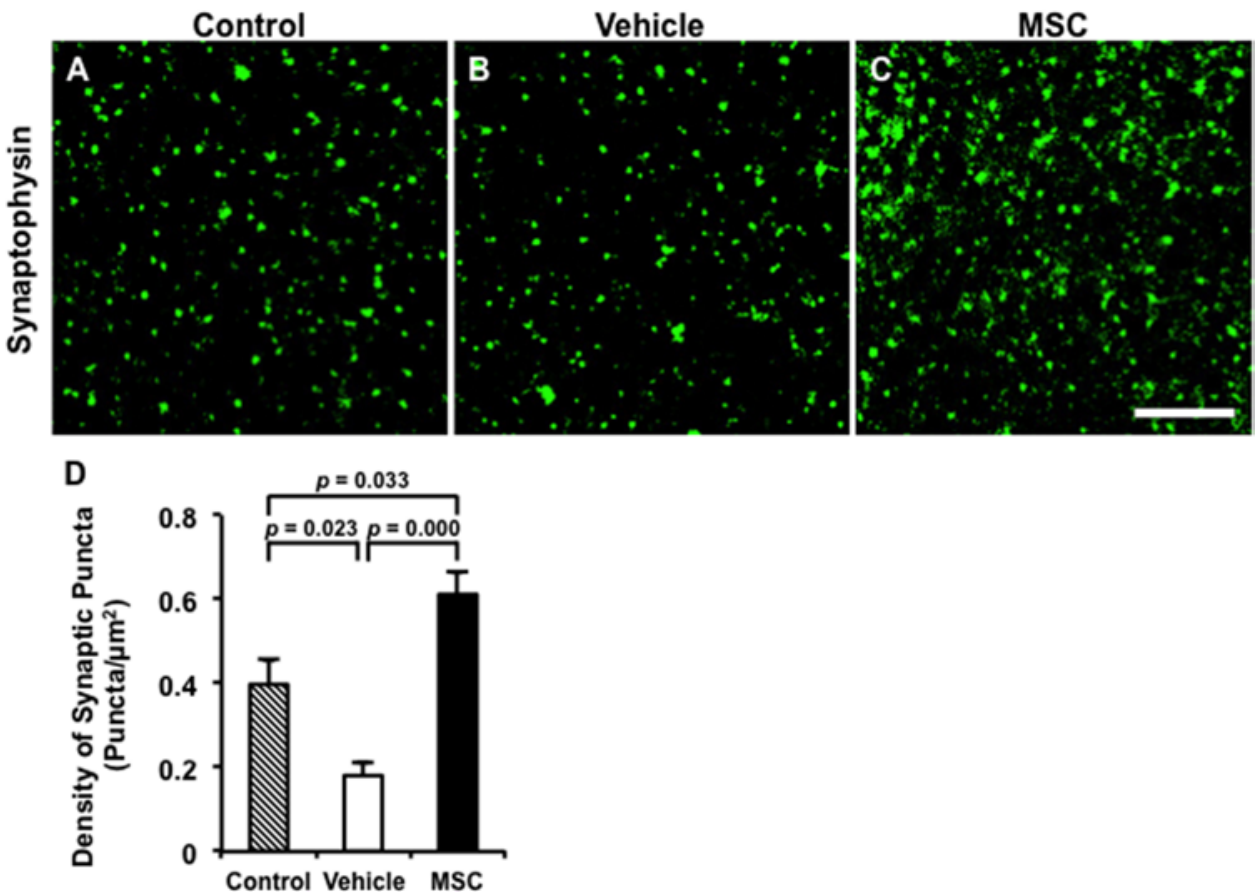

FIG. 6. Synaptogenesis was facilitated after the MSC intravenous infusions. Synaptogenesis was analyzed in the contralesional cortex at layer III/IV with confocal microscopy. Representative immunohistochemical images of the synaptophysin immunolabeling (green) in the control (A), vehicle (B), and MSC (C) groups are shown. The quantitative analysis of the density of the synaptic puncta showed that the MSC group had significantly increased puncta compared with the control and vehicle groups, while the control group had significantly increased puncta compared with the vehicle group. Bar $=5 \mu \mathrm{m}$.

"residual brain" volumes were greater in the MSC group than in the vehicle group. These results suggest that the intravenous infusion of MSCs facilitated the growth of brain tissue in the unaffected brain regions, even though the volumes of the ischemic regions that were damaged by the neonatal hypoxia did not differ between the groups at P35. The enlargement of unaffected brain tissue might contribute to the functional recovery observed after the intravenous infusions of MSCs in the HI model.

Histological examination confirmed brain growth in the contralesional hemisphere. Staining with hematoxylin and eosin revealed the increased thickness of both the contralesional cortex and corpus callosum. The number of $\mathrm{NeuN}^{+}$neurons in the MSC group was increased compared to the number in the vehicle group. We also observed an increase in the number of synaptic puncta that were immunostained for synaptophysin in the contralesional cortex, which suggests that neuronal compensation had occurred through the facilitation of brain growth by the intravenous infusion of MSCs.

The mechanisms underlying the functional recovery observed in HI have not been fully elucidated. However, previous studies have shown enhanced neuroplasticity in the contralesional hemisphere in spontaneous functional recovery. ${ }^{6}$ We do not expect that such large levels of neurogenesis and synaptic plasticity would occur in the adult brain. The striking changes in the nonlesional hemisphere observed here may relate to the inherent plasticity of the neonatal brain, which may render it more responsive to the therapeutic effects of MSCs. The developing brain is more malleable to external stimuli, including ischemia, compared to the adult brain, and this is generally considered advantageous with respect to functional recovery. ${ }^{19}$ In the present study, we observed that the intravenously infused GFP-MSCs were distributed in the contralesional cortex, and this might have been facilitated by signaling by trophic factors to cortical neurons that enhanced neural plasticity in this model. ${ }^{17}$ These results, in combination with the fact that the animals that received intravenous infusions of MSCs did not show epilepsy or other abnormal behaviors, suggest that the intravenous infusion of MSCs was effective and safe in this $\mathrm{HI}$ model.

We previously studied the effects of intravenous infusion of MSCs in a rat model of status epilepticus and
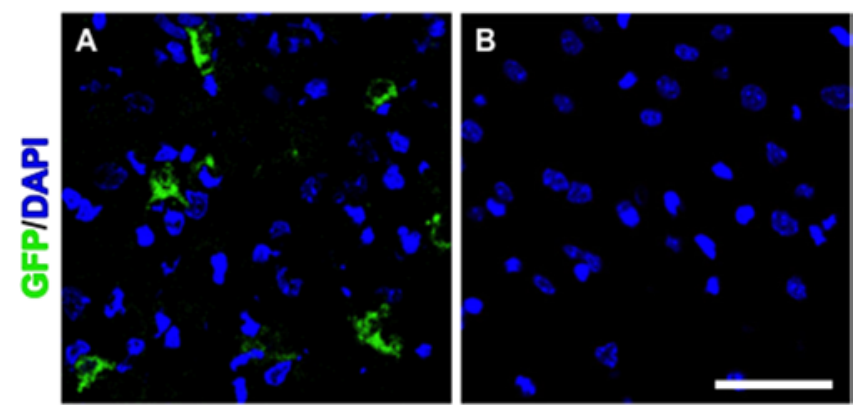

FIG. 7. Distribution of GFP-MSCs in the MSC-treated animals. The intravenously administered GFP-MSCs (green) were observed in the DAPI (blue)-counterstained contralesional cortex (A) in specimens obtained 3 days after the infusion. The confocal images show no $\mathrm{GFP}^{+}$signals in the non-GFP-MSC group (B). Bar $=50 \mu \mathrm{m}$. 
suggested based on the results that this therapy would be attractive for patients suffering from epilepsy after acute encephalitis. ${ }^{3}$ Similarly, we believe that the clinical application of autologous MSC therapy by intravenous administration holds promise for patients suffering from $\mathrm{CP}$ after hypoxic-ischemic encephalopathy.

\section{Conclusions}

In summary, the results presented here indicate that the intravenous infusion of MSCs improved motor function in a rat model of HI. The possible mechanisms for the results that we observed include the distribution of the infused MSCs to the cortex, where they may contribute to neural compensation through the facilitation of neurogenesis and synaptogenesis primarily in the nonlesional hemisphere.

\section{Acknowledgments}

This work was supported in part by JSPS KAKENHI grant number 26462168 (to T.S.), Merit Review Award 1 I01 BX003190 from the U.S. Department of Veterans Affairs Biomedical Laboratory Research and Development Service and the Rehabilitation and Research and Development Service (to J.D.K.). We are grateful to the National BioResource Project-Rat (http://www.anim.med. kyoto-u.ac.jp/NBR/) for providing this strain of rat [NBRP Rat No: 0273, W-Tg (CAG-GFP)184Ys].

\section{References}

1. de Paula S, Greggio S, Marinowic DR, Machado DC, DaCosta JC: The dose-response effect of acute intravenous transplantation of human umbilical cord blood cells on brain damage and spatial memory deficits in neonatal hypoxiaischemia. Neuroscience 210:431-441, 2012

2. de Paula S, Vitola AS, Greggio S, de Paula D, Mello PB, Lubianca JM, et al: Hemispheric brain injury and behavioral deficits induced by severe neonatal hypoxia-ischemia in rats are not attenuated by intravenous administration of human umbilical cord blood cells. Pediatr Res 65:631-635, 2009

3. Fukumura S, Sasaki M, Kataoka-Sasaki Y, Oka S, Nakazaki M, Nagahama H, et al: Intravenous infusion of mesenchymal stem cells reduces epileptogenesis in a rat model of status epilepticus. Epilepsy Res 141:56-63, 2018

4. Honma T, Honmou O, Iihoshi S, Harada K, Houkin K, Hamada $\mathrm{H}$, et al: Intravenous infusion of immortalized human mesenchymal stem cells protects against injury in a cerebral ischemia model in adult rat. Exp Neurol 199:56-66, 2006

5. Jellema RK, Wolfs TG, Lima Passos V, Zwanenburg A, Ophelders DR, Kuypers E, et al: Mesenchymal stem cells induce $\mathrm{T}$-cell tolerance and protect the preterm brain after global hypoxia-ischemia. PLoS One 8:e73031, 2013

6. Jung WB, Im GH, Chung JJ, Ahn SY, Jeon TY, Chang YS, et al: Neuroplasticity for spontaneous functional recovery after neonatal hypoxic ischemic brain injury in rats observed by functional MRI and diffusion tensor imaging. Neuroimage 126:140-150, 2016

7. Kim S, Honmou O, Kato K, Nonaka T, Houkin K, Hamada $\mathrm{H}$, et al: Neural differentiation potential of peripheral blood- and bone-marrow-derived precursor cells. Brain Res 1123:27-33, 2006

8. Komatsu K, Honmou O, Suzuki J, Houkin K, Hamada H, Kocsis JD: Therapeutic time window of mesenchymal stem cells derived from bone marrow after cerebral ischemia. Brain Res 1334:84-92, 2010

9. Lämmer AB, Beck A, Grummich B, Förschler A, Krügel $\mathrm{T}$, Kahn T, et al: The $\mathrm{P} 2$ receptor antagonist PPADS sup- ports recovery from experimental stroke in vivo. PLoS One 6:e19983, 2011

10. Matsuda Y, Sasaki M, Kataoka-Sasaki Y, Takayanagi A, Kobayashi K, Oka S, et al: Intravenous infusion of bone marrow-derived mesenchymal stem cells reduces erectile dysfunction following cavernous nerve injury in rats. Sex Med 6:49-57, 2018

11. Monbaliu E, Himmelmann K, Lin JP, Ortibus E, Bonouvrié $\mathrm{L}$, Feys H, et al: Clinical presentation and management of dyskinetic cerebral palsy. Lancet Neurol 16:741-749, 2017

12. Morita T, Sasaki M, Kataoka-Sasaki Y, Nakazaki M, Nagahama $\mathrm{H}$, Oka S, et al: Intravenous infusion of mesenchymal stem cells promotes functional recovery in a model of chronic spinal cord injury. Neuroscience 335:221-231, 2016

13. Murphy TH, Corbett D: Plasticity during stroke recovery: from synapse to behaviour. Nat Rev Neurosci 10:861-872, 2009

14. Nakamura H, Sasaki Y, Sasaki M, Kataoka-Sasaki Y, Oka $\mathrm{S}$, Nakazaki M, et al: Elevated brain derived neurotrophic factor (BDNF) levels in plasma but not serum reflect in vivo functional viability of infused mesenchymal stem cells after middle cerebral artery occlusion in rat. J Neurosurg Sci [epub ahead of print], 2017

15. Nakazaki M, Sasaki M, Kataoka-Sasaki Y, Oka S, Namioka $\mathrm{T}$, Namioka A, et al: Intravenous infusion of mesenchymal stem cells inhibits intracranial hemorrhage after recombinant tissue plasminogen activator therapy for transient middle cerebral artery occlusion in rats. J Neurosurg 127:917-926, 2017

16. Paxinos G, Watson C: The Rat Brain in Stereotaxic Coordinates, ed 6. Amsterdam: Academic Press, 2007

17. Ren G, Chen X, Dong F, Li W, Ren X, Zhang Y, et al: Concise review: mesenchymal stem cells and translational medicine: emerging issues. Stem Cells Transl Med 1:51-58, 2012

18. Rice JE III, Vannucci RC, Brierley JB: The influence of immaturity on hypoxic-ischemic brain damage in the rat. Ann Neurol 9:131-141, 1981

19. Rocha-Ferreira E, Hristova M: Plasticity in the neonatal brain following hypoxic-ischaemic injury. Neural Plast 2016:4901014, 2016

20. Rumajogee P, Bregman T, Miller SP, Yager JY, Fehlings MG: Rodent hypoxia-ischemia models for cerebral palsy research: a systematic review. Front Neurol 7:57, 2016

21. Sasaki Y, Sasaki M, Kataoka-Sasaki Y, Nakazaki M, Nagahama H, Suzuki J, et al: Synergic effects of rehabilitation and intravenous infusion of mesenchymal stem cells after stroke in rats. Phys Ther 96:1791-1798, 2016

22. Smith AL, Alexander M, Rosenkrantz TS, Sadek ML, Fitch $\mathrm{RH}$ : Sex differences in behavioral outcome following neonatal hypoxia ischemia: insights from a clinical meta-analysis and a rodent model of induced hypoxic ischemic brain injury. Exp Neurol 254:54-67, 2014

23. Smith AL, Rosenkrantz TS, Fitch RH: Effects of sex and mild intrainsult hypothermia on neuropathology and neural reorganization following neonatal hypoxic ischemic brain injury in rats. Neural Plast 2016:2585230, 2016

24. Stavsky M, Mor O, Mastrolia SA, Greenbaum S, Than NG, Erez O: Cerebral palsy-trends in epidemiology and recent development in prenatal mechanisms of disease, treatment, and prevention. Front Pediatr 5:21, 2017

25. West MJ: New stereological methods for counting neurons. Neurobiol Aging 14:275-285, 1993

26. Yasuhara T, Hara K, Maki M, Mays RW, Deans RJ, Hess DC, et al: Intravenous grafts recapitulate the neurorestoration afforded by intracerebrally delivered multipotent adult progenitor cells in neonatal hypoxic-ischemic rats. J Cereb Blood Flow Metab 28: 1804-1810, 2008

27. Yasuhara T, Hara K, Maki M, Xu L, Yu G, Ali MM, et al: 
Mannitol facilitates neurotrophic factor up-regulation and behavioural recovery in neonatal hypoxic-ischaemic rats with human umbilical cord blood grafts. J Cell Mol Med 14:914921, 2010

\section{Disclosures}

The authors report no conflict of interest concerning the materials or methods used in this study or the findings specified in this paper.

\section{Author Contributions}

Conception and design: Sasaki, Sakai, Kataoka-Sasaki, Fukumura, Tsutsumi, Kocsis, Honmou. Acquisition of data: Sakai. Analy- sis and interpretation of data: Sasaki, Sakai, Kataoka-Sasaki, Oka, Kocsis, Honmou. Drafting the article: Sasaki, Sakai, Kocsis, Honmou. Critically revising the article: all authors. Reviewed submitted version of manuscript: all authors. Approved the final version of the manuscript on behalf of all authors: Sasaki. Statistical analysis: Sakai, Nakazaki. Administrative/technical/material support: Sasaki, Kobayashi. Study supervision: Sasaki, KataokaSasaki, Oka, Fukumura, Kobayashi, Tsutsumi, Kocsis, Honmou.

\section{Correspondence}

Masanori Sasaki: Sapporo Medical University School of Medicine, Sapporo, Hokkaido, Japan.msasaki@sapmed.ac.jp. 